\title{
Economic evaluation of alternative testing regimes and settings to detect undiagnosed HIV in Australia
}

\author{
Owain D. Williams ${ }^{1}$, Judith A. Dean ${ }^{1}$, Anna Crothers ${ }^{2}$, Charles F. Gilks ${ }^{1}$ and Jeff Gow G $^{3,4^{*}}$ (I)
}

\begin{abstract}
Background: The study aimed to estimate the comparative costs per positive diagnosis of previously undetected HIV in three testing regimes: conventional; parallel and point of care (POC) testing. The regimes are analysed in six testing settings in Australia where infection is concentrated but with low prevalence.

Methods: A cost model was developed to highlight the trade-offs between test and economic efficiency from a provider perspective. First, an estimate of the number of tests needed to find a true (previously undiagnosed) positive diagnosis was made. Second, estimates of the average cost per positive diagnosis in whole of population (WoP) and men who have sex with men (MSM) was made, then third, aggregated to the total cost for diagnosis of all undetected infections.

Results: Parallel testing is as effective as conventional testing, but more economically efficient. POC testing provide two significant advantages over conventional testing: they screen out negatives effectively at comparatively lower cost and, with confirmatory testing of reactive results, there is no loss in efficiency. The average and total costs per detection in WoP are prohibitive, except for Home Self Testing. The diagnosis in MSM is cost effective in all settings, but especially using Home Self Testing when the individual assumes the cost of testing.

Conclusions: This study illustrates the trade-offs between economic and test efficiency and their interactions with population(s) prevalence. The efficient testing regimes and settings are presently under or not funded in Australia. Home Self Testing has the potential to dramatically increase testing rates at very little cost.
\end{abstract}

Keywords: HIV testing, Conventional testing, Parallel testing, Point of care testing, Self-testing, Costs

\section{Background}

Ambitious 90-90-90 targets have been set to diagnose 90\% of all people living with HIV (PLHIV), initiate antiretroviral treatment (ART) for $90 \%$ of those diagnosed with HIV infection and to achieve an undetectable viral load in $90 \%$ of those on ART [1]. To achieve the first 90 , increased levels of testing needs to occur. Of course,

\footnotetext{
* Correspondence: gowj@usq.edu.au

${ }^{3}$ School of Commerce, University of Southern Queensland, Toowoomba, Queensland 4350, Australia

${ }^{4}$ School of Accounting, Economics and Finance, University of KwaZulu-Natal, Durban, South Africa

Full list of author information is available at the end of the article
}

dynamically, as incidence falls over time due to reduced rates of transmission, the cost of detecting the last $10 \%$ of prevalence will increase. The 'last mile problem' is generic to infectious disease elimination campaigns [2] especially with regard to the incremental costeffectiveness (and incrementally elevated costs) of targeted screening that necessarily turns its focus to more hard to reach populations and infrequent or non-testers. In disease elimination strategies, the incremental costs of screening and diagnosis can be expected to rise as prevalence falls, and thus the cost-effectiveness of all available means of testing becomes a compelling area of 
investigation by which to gear resources and public health interventions.

The HIV prevalence in Australia among adults aged 15 years and older is estimated to be $0.14 \%$ in 2018 [3]. Transmission of HIV in Australia continues to occur primarily through male-to-male sexual contact (63\% of all infections) resulting in a concentrated epidemic among men who have sex with men (MSM) (prevalence of 7.9\%) [3]. In 2017, estimates suggest that 2899 (11\%) people living with HIV were undiagnosed and among HIV positive MSM, 9\% were undiagnosed [3]. Australia, therefore, needs to explore and consider cost effective means of detecting the last $10 \%$.

In this context, this study aims to analyse the costs of making a HIV positive diagnosis using the three main testing regimes available electively in Queensland, Australia: conventional; parallel; and point of care (POC) testing; in six common testing settings: Private General Practice (GP); Public Sexual Health Clinic (SHC); Community Peer Organisation led General Practice (Community GP); Community Organisation led Volunteer Peer Clinic; Community Organisation led Salaried Peer Clinic; and Private at Home. The study did not consider testing in hospital settings or screening (blood and organ safety).

\section{Methods}

\section{Aim}

A model was developed to compare costs from the service provider perspective and to illustrate the trade-offs between test effectiveness (mainly arising from the sensitivity of the test technologies) and the economic efficiency associated with the application of these different regimes in six different testing settings.

The model seeks to demonstrate how configurations of testing technologies, regimes and settings and patient pathways can be displayed in a manner that is more informative than those generated by decision tree models. A further objective is also to generate a relatively simple model (or framework) that accommodates the sensitivities of the testing technologies used in each of three testing regimes and six settings in combination with the rates of prevalence and incidence in the whole of population (WoP) and the priority population of men who have sex with men (MSM).

\section{HIV testing regimes}

There are three regimes that are most commonly used for elective HIV testing/screening in Australia: Conventional EIA (enzyme-linked immunoassay) laboratory testing; Parallel testing; and POC testing including HIV self-testing (HIVST). The diagnostic standard in Australia requires the reactive/positive result of the initial screening test to be confirmed by a laboratory based confirmatory Western Blot (WB) or additional supplementary blood test. Establishing a true positive diagnosis involves individuals entering one of three HIV testing regimes. Each involves various different combinations of initial and confirmatory testing technologies as part of the pathway to confirmed diagnosis.

The conventional regime is mainly used in GP clinics where the vast majority of testing for HIV and other sexually transmissible infections (STI) now occurs and in SHC [4].

In parallel testing both conventional EIA testing and rapid POC testing (DHC) are performed simultaneously at the point of testing by a health professional or appropriately trained testing facilitator $[5,6]$. Parallel testing can increase the number of tests used in low prevalence countries compared to conventional testing. However, it is efficient as it enables results to be concordantly confirmed by two different initial assays at one point of service [7], thus reducing the need to wait for results or for return visits, both identified as barriers to HIV testing [8-11].

The POC testing regime allows testing to occur in non-clinical settings by medical and non-medical personnel with results available at the time and place of testing or at home as is the case with HIVST [5, 12]. The rapid POC tests do not provide confirmatory diagnostic evidence of a HIV infection; and patients with reactive results require confirmation of a positive result by entering into the conventional or parallel testing regimes. The three regimes are represented diagrammatically in Fig. 1.

\section{HIV testing settings}

All six settings were based in Brisbane, the capital city in the Australian state of Queensland, which are representative of the whole of Australia. Conventional testing pathway is conducted in SHC and Private GP setting. Parallel testing pathway is mainly available at community GP clinics, community volunteer peer clinics and community salaried peer clinics. POC testing pathway included both rapid POC testings and HIVST.

\section{Cost model}

A cost model was constructed in Excel to compare the cost per positive diagnosis associated with two populations, three regimes and six settings and incorporating a number of inputs. Each testing regime can result in a number of possible outcomes.

For example, conventional testing could produce four possible outcomes: a true positive, a true negative, a false positive, or a false negative. Identifying all possible outcomes enables the calculation of the total costs of each outcome, the likelihood of each of these outcomes and the calculation of the average cost per person tested. 


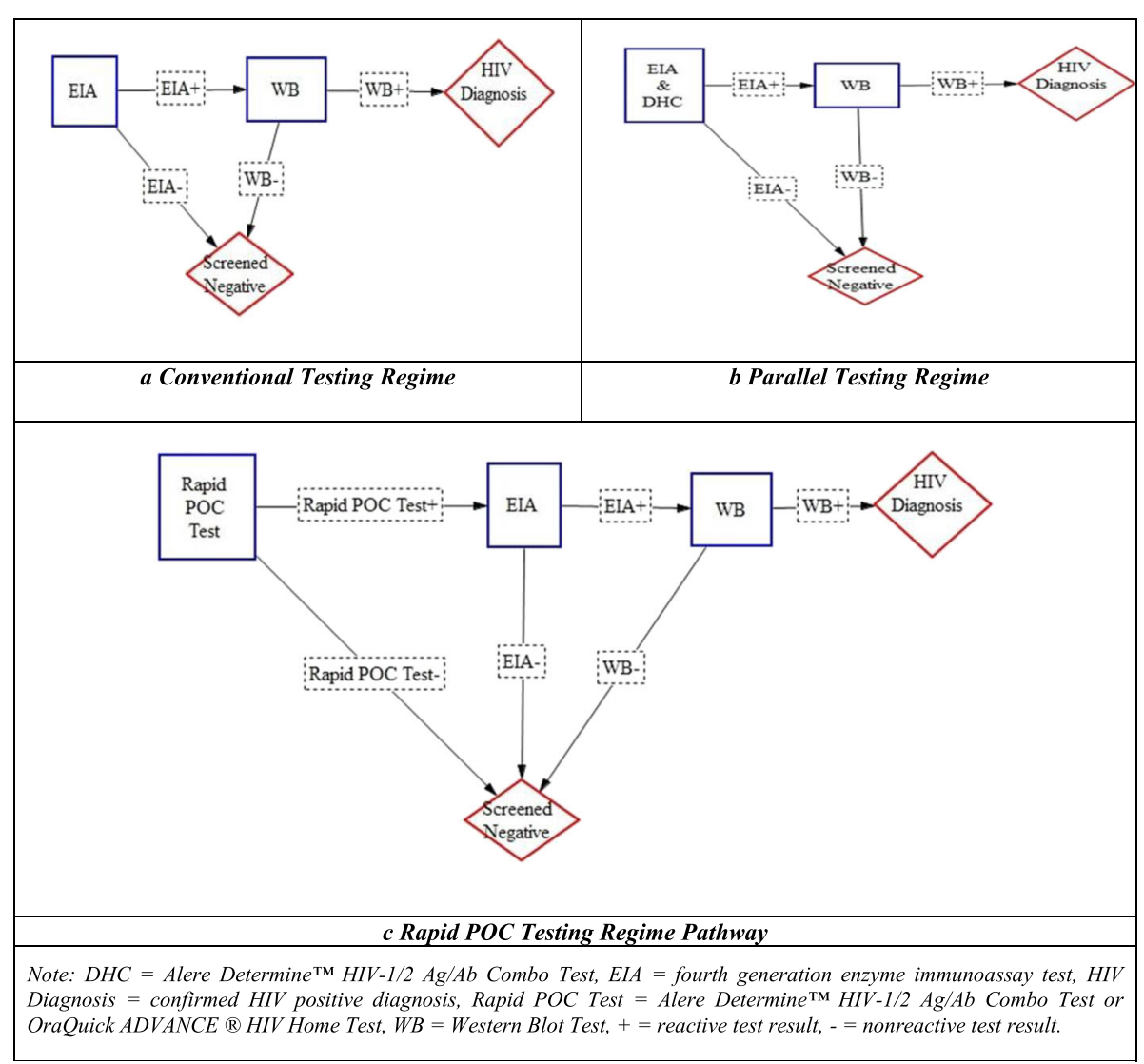

Fig. 1 HIV Testing Regime Pathways to Confirmed Diagnosis. Note: DHC = Alere Determine ${ }^{\mathrm{TM}} \mathrm{HIV}-1 / 2 \mathrm{Ag} / \mathrm{Ab}$ Combo Test, EIA = fourth generation enzyme immunoassay test, HIV Diagnosis = confirmed HIV positive diagnosis, Rapid POC Test = Alere Determine ${ }^{\mathrm{TM}} \mathrm{HIV}-1 / 2 \mathrm{Ag} / \mathrm{Ab}$ Combo Test or OraQuick ADVANCE ${ }^{-}$HIV Home Test, WB $=$Western Blot Test, $+=$reactive test result, $-=$nonreactive test result

The probability of each outcome is dependent on three main model inputs: first, the probability of finding an undiagnosed HIV infection in both whole of population (WoP) and MSM; second, the sensitivity (Sen) and specificity (Spec) of each testing technology; and third, the cost inputs for each testing regime.

For example, the average cost per person tested for the conventional testing regime is calculated as:

$$
\begin{aligned}
& =\left(P_{H I V} * \operatorname{Sen}_{E I A} * \operatorname{Sen}_{W B}\right) * A C \text { per True HIV Positive } E_{E I A+, W B+} \\
& +\left(P_{H I V} *\left(1-\text { Sen }_{E I A}\right)\right) * A C \text { per False HIV Negative } \text { EIA }_{-} \\
& +\left(\left(1-P_{H I V}\right) * \operatorname{Spec}_{E I A}\right) * A C \text { per True HIV negative } \text { EIA }_{-} \\
& +\left(\left(1-P_{H I V}\right) *\left(1-\operatorname{Spec}_{E I A}\right) * \operatorname{Spec}_{W B}\right) * A C \text { per False HIV Positive } \text { EIA }_{\text {,WB }}
\end{aligned}
$$

Then the average number of tests needed to find a positive and previously undiagnosed HIV case for the conventional testing is: $=\frac{1}{P_{H I V} * \operatorname{Sen}_{\mathrm{EIA}} * \operatorname{Sen}_{W B} \text {. }}$

The average cost per positive diagnosis is derived as:

$=$ Average Cost per person tested $*$ Average number of tests required for a positive diagnosis

Details and assumptions for the different screening strategies modelled are presented below. The test efficiency forms part of the calculation of the probability of a finding a true undiagnosed case in each of the two populations. This factor provides a benchmark to evaluate and compare the economic efficiency of each of the three regimes and six settings.

$$
\begin{aligned}
& \text { Rate of undiagnosed HIV cases in population } \\
& =\frac{1}{P_{H I V}}
\end{aligned}
$$

\section{Data}

\section{Clinical test effectiveness}

The following testing technologies and documented sensitivities and specificities were used for calculations: Architect HIV Ag/Ab Combo assay which is generally used in Australia for conventional testing [Sensitivity 99.94\% (95\% CI, 99.79, 100\%); Specificity 99.50\% (95\% CI, 99.31, 99.64)] [13]; Rapid Alere Determine ${ }^{\text {mu }}$ Combo widely used in Queensland for POC testing [Sensitivity 99.4\% (95\% CI, 96.6, 100\%); Specificity 99.2\% (95\% CI, 98.2, 99.7)] [14]; and the OraQuick ADVANCE ${ }^{\bullet}$ HIV 
Home Test for self-testing as this particular kit is the closest to market approval in Australia [Sensitivity 91.67\% (95\% CI, 84.24, 96.33\%); Specificity 99.98\% (95\% CI, 99.89, 100)] [15].

\section{Probability of detecting an undiagnosed HIV infection}

The probability of detection is derived by deduction from the estimates of the number of undiagnosed HIV positive cases.

Probability of finding an undiagnosed HIV case in population ${ }_{i}$ $=\frac{\text { Number of undiagnosed HIV cases in population }_{\mathrm{i}}}{\text { population }_{\mathrm{i}} \text { size }}$

In 2014, the Australian WoP aged 15 years or greater was 18,754,954 [16]. The MSM population was estimated at 305,783 using data from the 2nd Australian Study of Health and Relationships Survey [17] and demographic information from the Australian Bureau of Statistics [16]. It should be noted that there are limited data available on the relative size and distribution of the MSM population in the states and territories of Australia, and any enumeration of this population at the sub-national level is complex and subject to certain assumptions. Thus, there is a high degree of uncertainty in these estimates.
Data on HIV incidence and estimated undiagnosed prevalence for both the WoP and MSM populations was derived from the Australia Annual HIV Surveillance Report published in 2015 [18]. National rates of undiagnosed HIV prevalence were used because only national population estimates for undiagnosed prevalence in the general and MSM population are available. Additionally, the latest survey data reports only national prevalence of the number of men who identify as MSM. The number of undiagnosed HIV positive people in the general population in 2014 was estimated to be 3350 (CI 95\%, 21004670) and in the MSM population 1848 (CI 95\%, 8502896). Thus, mass universal testing in WoP would yield a $0.0179 \%$ probability of finding an undiagnosed HIV infection per person tested and a $0.6044 \%$ probability in the MSM population assuming everyone has an equal chance of being tested.

\section{Cost data collection}

From February to April 2016 costing information was collected from community sites, SHC and GP clinics. Costs incurred by the health system include: consult length; staff salary; consult costs including Medicare

Table 1 Conventional Testing Regime: Private General Practice Clinic Setting - (Funded by Federal Government - Medicare)

\begin{tabular}{|c|c|c|c|c|}
\hline \multirow[b]{2}{*}{ Outcomes } & \multicolumn{2}{|c|}{$\begin{array}{l}\text { Probability of True HIV Positive } \\
\left(P_{H I V}\right)\end{array}$} & \multicolumn{2}{|c|}{$\begin{array}{l}\text { Probability of True HIV Negative } \\
\left(1-P_{H I V}\right)\end{array}$} \\
\hline & $\begin{array}{l}\text { HIV Positive } \\
(E I A+\& W B+)\end{array}$ & $\begin{array}{l}\text { False HIV } \\
\text { Negative } \\
\text { (EIA-) }\end{array}$ & $\begin{array}{l}\text { False HIV Positive } \\
\text { (EIA+ \& WB-) }\end{array}$ & $\begin{array}{l}\text { True HIV } \\
\text { Negative } \\
\text { (EIA-) }\end{array}$ \\
\hline Probability of result & $\begin{array}{l}\left(P_{H I V} * \operatorname{Sen}_{E I A} *\right. \\
\left.\operatorname{Sen}_{W B}\right)\end{array}$ & $P_{H I V} *\left(1-\operatorname{Sen}_{E I A}\right)$ & $\begin{array}{l}\left(\left(1-P_{H I V}\right) *\left(1-\operatorname{Spec}_{E I A}\right) *\right. \\
\operatorname{Spec}_{W B}\end{array}$ & $\begin{array}{l}\left(1-P_{H I V}\right) * \\
\operatorname{Spec}_{E I A}\end{array}$ \\
\hline \multicolumn{5}{|l|}{ Inputs } \\
\hline \multicolumn{5}{|l|}{ Visit 1: Initial Screening } \\
\hline Consult time & $20 \mathrm{~min}$ & $20 \mathrm{~min}$ & $20 \mathrm{~min}$ & $20 \mathrm{~min}$ \\
\hline Medicate Rebate & $\$ 71.70^{1}$ & $\$ 71.70^{1}$ & $\$ 71.70^{1}$ & $\$ 71.70^{1}$ \\
\hline Staffing & GP & GP & GP & GP \\
\hline \multicolumn{5}{|l|}{ Pathology costs } \\
\hline EIA & $\$ 15.65^{2}$ & $\$ 15.65^{2}$ & $\$ 15.65^{2}$ & $\$ 15.65^{2}$ \\
\hline WB confirmatory & $\$ 29.00^{3}$ & & $\$ 29.00^{3}$ & \\
\hline \multicolumn{5}{|l|}{ Visit 2: Test Results } \\
\hline Consult time & $40 \mathrm{~min}$ & $20 \mathrm{~min}$ & $20 \mathrm{~min}$ & $20 \mathrm{~min}$ \\
\hline Medicate Rebate & $\$ 105.55^{4}$ & $\$ 71.70^{1}$ & $\$ 71.70^{1}$ & $\$ 71.70^{1}$ \\
\hline Staffing & GP & GP & GP & GP \\
\hline Total Cost of Each Outcome & $\$ 221.90$ & $\$ 159.05$ & $\$ 188.05$ & $\$ 159.05$ \\
\hline Average cost $(A C)$ per person tested & \multicolumn{4}{|c|}{$\begin{array}{l}=\left(P_{H I V} * \operatorname{Sen}_{E I A} * \operatorname{Sen}_{W B}\right) * A C \text { per True HIV Positive } e_{E I A+}, W_{B+}+\left(P_{H I V} *\left(1-\operatorname{Sen}_{E I A}\right)\right) * \\
\text { AC per False HIV Negative } e_{E I A-}+\left(\left(1-P_{H I N}\right) * \operatorname{SpeC}_{E I A}\right) * A C \text { per True HIV Negative } E_{E I A-}+\left(\left(1-P_{H I V}\right) *(1-\right. \\
\left.\left.\left.\operatorname{Spec}_{E I A}\right) * \operatorname{Spec}_{W B}\right)\right) * A C \text { per False HIV Positive }\end{array}$} \\
\hline $\begin{array}{l}\text { Average number of tests for a positive } \\
\text { diagnosis }\end{array}$ & \multicolumn{4}{|l|}{$=\frac{1}{P_{H I N} * \operatorname{Sen}_{E A} * \operatorname{Sen}_{W B}}$} \\
\hline
\end{tabular}

Note: AC Average Cost, EIA fourth generation enzyme immunoassay test, GP General Practitioner, P Probability, Sen Sensitivity, Spec Specificity, WB Western Blot Test, + reactive test result, - nonreactive test result

1 = Medicare Rebate Item 36; 2 = Medicare Rebate Item 69,384; 3 = Medicare Rebate Item 69,387; 4 = Medicare Rebate Item 44 
Benefits Schedule rebates from the publicly funded universal health care system operated by the Commonwealth of Australia Department of Human Services; test costs; and confirmatory laboratory testing for reactive results. Costs were calculated from the health care provider's perspective, with the exception of HIVST, which was based on the retail price of the test kit established elsewhere [19].

The cost of testing in the six settings and by HIV status are outlined in Tables 1, 2, 3, 4, 5 and 6.

\section{Results}

\section{Test efficiency in detecting undiagnosed HIV cases}

Conventional and parallel testing regimes were found to be more efficient (higher sensitivity and specificity) in detecting a confirmed positive HIV diagnosis than POC testing, resulting in the number of tests required to detect HIV being lower for the former regimes compared to the latter (Tables A1 to A6b in Additional file 1). Conventional, parallel and Point of Care testing conducted at a community organisation each detect undiagnosed cases at an approximate rate of one per 5602 and one per 5787 per person tested respectively, and in WoP at 165 and 171, respectively in the MSM population. In comparison, home Self Testing results in slightly higher rates at one per 6111 per person in the WoP and one per 180 in the MSM population.

\section{Costs}

Costs vary across regimes and settings depending on the number of visits, labour costs and types of labour used, and the number (if any) of pathology tests required.

The estimated cost per HIV diagnosis in the six settings for WoP were found to be extremely high, except for HIVST, when private costs were ignored (Table 7). Secondly, the cost per diagnosis for the MSM population were considerably lower due to the higher prevalence and therefore ease of detection, compared to WoP (Table 8).

The results of this study demonstrate that both test effectiveness and economic efficiency are constituents in comparing cost per HIV diagnosis in all testing settings. First, it is clear that clinicians' wages are significant drivers of the costs of individual HIV testing and its detection. Conventional testing with clinical nurses and parallel testing with volunteer peer testers are three times more cost effective than either regime with GP/ doctor's wages. Parallel testing saves costs compared to

Table 2 Conventional Testing Regime: Public Sexual Health Clinic Setting (Funded by State Government)

\begin{tabular}{|c|c|c|c|c|}
\hline \multirow[b]{2}{*}{ Outcomes } & \multicolumn{2}{|c|}{ True HIV Positive $\left(P_{H I V}\right)$} & \multicolumn{2}{|l|}{ True HIV Negative $\left(1-P_{H I V}\right)$} \\
\hline & $\begin{array}{l}\text { HIV Positive } \\
\text { (EIA+ \& WB+) }\end{array}$ & $\begin{array}{l}\text { False HIV } \\
\text { Negative } \\
\text { (EIA-) }\end{array}$ & $\begin{array}{l}\text { False HIV Positive } \\
\text { (EIA+ \& WB-) }\end{array}$ & $\begin{array}{l}\text { True HIV } \\
\text { Negative } \\
\text { (EIA-) }\end{array}$ \\
\hline Probability of result & $\begin{array}{l}\left(P_{H I V} * \operatorname{Sen}_{E I A} *\right. \\
\left.\operatorname{Sen}_{W B}\right)\end{array}$ & $\begin{array}{l}\left(P_{H I V} *(1-\right. \\
\left.\left.\operatorname{Sen}_{E I A}\right)\right)\end{array}$ & $\begin{array}{l}\left(\left(1-P_{H I V}\right) *\left(1-S_{\text {Pec }} I A\right) *\right. \\
\left.\operatorname{Spec}_{W B}\right)\end{array}$ & $\begin{array}{l}\left(1-P_{H I V}\right) * \\
\operatorname{Spec}_{E I A}\end{array}$ \\
\hline \multicolumn{5}{|l|}{ Inputs } \\
\hline \multicolumn{5}{|l|}{ Visit 1: Initial Screening } \\
\hline Consult time & 30 Min & $30 \mathrm{Min}$ & $30 \mathrm{Min}$ & $30 \mathrm{Min}$ \\
\hline Staff Wage (Rate/Hour): & $\$ 45.12$ & $\$ 45.12$ & $\$ 45.12$ & $\$ 45.12$ \\
\hline Staffing & Clinical Nurse & Clinical Nurse & Clinical Nurse & Clinical Nurse \\
\hline \multicolumn{5}{|l|}{ Pathology costs } \\
\hline EIA & $\$ 18.54$ & $\$ 18.54$ & $\$ 18.54$ & $\$ 18.54$ \\
\hline WB confirmatory & $\$ 84.54$ & & $\$ 84.54$ & \\
\hline \multicolumn{5}{|l|}{ Visit 2: Test Results } \\
\hline Consult time & $60 \mathrm{Min}$ & $30 \mathrm{Min}$ & $30 \mathrm{Min}$ & $30 \mathrm{Min}$ \\
\hline Staff Wage (Rate/Hour): & $\$ 78.00$ & $\$ 45.12$ & $\$ 45.12$ & $\$ 45.12$ \\
\hline Staffing & $\begin{array}{l}\text { Staff specialist } \\
\text { (Level 18) }\end{array}$ & Clinical Nurse & Clinical Nurse & Clinical Nurse \\
\hline Total Cost of Each Outcome & $\$ 203.64$ & $\$ 63.67$ & $\$ 148.21$ & $\$ 63.67$ \\
\hline Average cost per person tested & \multicolumn{4}{|c|}{$\begin{array}{l}=\left(P_{H I V} * \operatorname{Sen}_{E I A} * \operatorname{Sen}_{W B}\right) * A C \text { per True HIV Positive } E_{E I A+}, \text { WB }_{+}+\left(P_{H I V} *\left(1-\operatorname{Sen}_{E I A}\right)\right) * \\
\text { AC per False HIV Negative } e_{E I A-}+\left(\left(1-P_{H I N}\right) * \operatorname{SpeC}_{E I A}\right) * A C \text { per True HIV Negative } e_{E I A-}+\left(\left(1-P_{H I V}\right) *(1-\right. \\
\left.\left.\left.\text { SpeC }_{E I A}\right) * \operatorname{SpeC}_{W B}\right)\right) * \text { AC per False HIV Positive } e_{E I A+}, \text { WB- }\end{array}$} \\
\hline $\begin{array}{l}\text { Average number of tests for a positive } \\
\text { diagnosis }\end{array}$ & \multicolumn{4}{|c|}{$=\frac{1}{P_{H I V} * \operatorname{Sen}_{E A} * \operatorname{Sen}_{W B}}$} \\
\hline
\end{tabular}

Note: $A C$ Average Cost, EIA fourth generation enzyme immunoassay test, $P$ Probability, Sen Sensitivity, Spec Specificity, WB Western Blot Test, + reactive test result, - nonreactive test result 
Table 3 Parallel Testing Regime: Community Peer Organisation Bulk Billing GP Clinic Setting (Funded by Federal Government Medicare and State Government)

\begin{tabular}{|c|c|c|c|c|c|c|c|c|}
\hline \multirow[b]{2}{*}{ Outcomes } & \multicolumn{4}{|c|}{ True HIV Positive $\left(P_{\text {HIV }}\right)$} & \multicolumn{4}{|c|}{ True HIV Negative $\left(1-P_{H I V}\right)$} \\
\hline & $\begin{array}{l}\mathrm{DHC}+ \\
\mathrm{EIA}+, \mathrm{WB}+\end{array}$ & $\begin{array}{l}\text { DHC-, ElA+, } \\
\text { WB+ }\end{array}$ & $\begin{array}{l}\text { DHC+, } \\
\text { EIA- }\end{array}$ & $\begin{array}{l}\text { DHC-, } \\
\text { EIA- }\end{array}$ & DHC+, EIA- & $\mathrm{DHC}+, \mathrm{EIA}+, \mathrm{WB}-$ & DHC-, EIA+,WB- & DHC-, EIA- \\
\hline $\begin{array}{l}\text { Probability of } \\
\text { result }\end{array}$ & $\begin{array}{l}\left(P_{H I V} *\right. \\
\operatorname{Sen}_{D H C} * \\
\operatorname{Sen}_{E I A} * \\
\left.\operatorname{Sen}_{W B}\right)\end{array}$ & $\begin{array}{l}\left(P_{H I V} *(1-\right. \\
\left.\operatorname{Sen}_{D H C}\right) * \\
\operatorname{Sen}_{E I A} * \\
\left.\operatorname{Sen}_{W B}\right)\end{array}$ & $\begin{array}{l}\left(P_{H I V} *\right. \\
\operatorname{Sen}_{D H C} \\
*(1- \\
\left.\left.\operatorname{Sen}_{E I A}\right)\right)\end{array}$ & $\begin{array}{l}\left(P_{H I V^{*}}\right. \\
(1- \\
\left.\operatorname{Sen}_{D H C}\right) \\
*(1- \\
\left.\left.\operatorname{Sen}_{E I A}\right)\right)\end{array}$ & $\begin{array}{l}\left(\left(1-P_{H I V}\right)\right. \\
*(1- \\
\left.\operatorname{Spec}_{D H C}\right) * \\
\left.\operatorname{Spec}_{E I A}\right)\end{array}$ & $\begin{array}{l}\left(\left(1-P_{H I V}\right) *(1-\right. \\
\left.\operatorname{Spec}_{D H C}\right) *(1- \\
\left.\left.\operatorname{Spec}_{E I A}\right) * \operatorname{Spec}_{W B}\right)\end{array}$ & $\begin{array}{l}\left(\left(1-P_{H I V}\right) *\right. \\
\operatorname{Spec}_{D H C} *(1- \\
\left.\operatorname{Spec}_{E I A}\right) * \\
\left.\operatorname{Spec}_{W B}\right)\end{array}$ & $\begin{array}{l}\left(\left(1-P_{H I V}\right) *\right. \\
\operatorname{Spec}_{D H C} * \\
\left.\operatorname{Spec}_{E I A}\right)\end{array}$ \\
\hline \multicolumn{9}{|l|}{ Inputs } \\
\hline \multicolumn{9}{|c|}{ Visit 1: Initial Screening } \\
\hline Consult time & $20 \mathrm{~min}$ & 20 min & $20 \mathrm{~min}$ & $20 \mathrm{~min}$ & $20 \mathrm{~min}$ & $20 \mathrm{~min}$ & $20 \mathrm{~min}$ & $20 \mathrm{~min}$ \\
\hline $\begin{array}{l}\text { Medicate } \\
\text { Rebate }\end{array}$ & $\$ 71.70^{1}$ & $\$ 71.70^{1}$ & $\$ 71.70^{1}$ & $\$ 71.70^{1}$ & $\$ 71.70^{1}$ & $\$ 71.70^{1}$ & $\$ 71.70^{1}$ & $\$ 71.70^{1}$ \\
\hline Staffing & GP & GP & GP & GP & GP & GP & GP & GP \\
\hline \multicolumn{9}{|l|}{ Pathology costs } \\
\hline $\begin{array}{l}\text { Rapid Alere } \\
\text { Determine Test } \\
\text { (DHC) }\end{array}$ & $\$ 11$ & $\$ 11$ & $\$ 11$ & $\$ 11$ & $\$ 11$ & $\$ 11$ & $\$ 11$ & $\$ 11$ \\
\hline EIA & $\$ 15.65^{2}$ & $\$ 15.65^{2}$ & $\$ 15.65^{2}$ & $\$ 15.65^{2}$ & $\$ 15.65^{2}$ & $\$ 15.65^{2}$ & $\$ 15.65^{2}$ & $\$ 15.65^{2}$ \\
\hline $\begin{array}{l}\text { WB } \\
\text { confirmatory }\end{array}$ & $\$ 29.00^{3}$ & $\$ 29.00^{3}$ & & & & $\$ 29.00^{3}$ & $\$ 29.00^{3}$ & \\
\hline \multicolumn{9}{|c|}{ Visit 2: Test Results } \\
\hline Consult time & $40 \mathrm{~min}$ & $40 \mathrm{~min}$ & $20 \mathrm{~min}$ & & $20 \mathrm{~min}$ & $20 \mathrm{~min}$ & $20 \mathrm{~min}$ & \\
\hline $\begin{array}{l}\text { Medicare } \\
\text { Rebate }\end{array}$ & $\$ 105.55^{4}$ & $\$ 105.55^{4}$ & $\$ 71.70^{1}$ & & $\$ 71.70^{1}$ & $\$ 71.70^{1}$ & $\$ 71.70^{1}$ & \\
\hline Staffing & GP & GP & GP & & GP & GP & GP & \\
\hline $\begin{array}{l}\text { Total Cost of Each } \\
\text { Outcome }\end{array}$ & $\$ 232.90$ & $\$ 232.90$ & $\$ 170.05$ & $\$ 98.35$ & $\$ 170.05$ & $\$ 199.05$ & $\$ 199.05$ & $\$ 98.35$ \\
\hline $\begin{array}{l}\text { Average cost per } \\
\text { person tested }\end{array}$ & \multicolumn{8}{|c|}{ 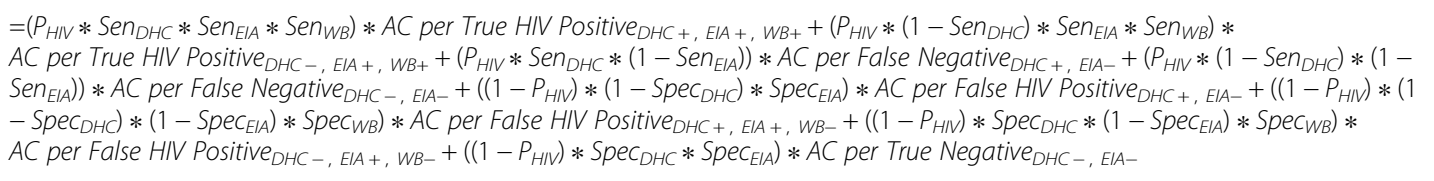 } \\
\hline $\begin{array}{l}\text { Average number } \\
\text { of tests for a } \\
\text { positive diagnosis }\end{array}$ & \multicolumn{8}{|c|}{$=\frac{1}{\left(P_{H N} * \operatorname{Sen}_{D H C} * \operatorname{Sen}_{E A} * \operatorname{Sen}_{W B}\right)+\left(P_{H V} *\left(1-\operatorname{Sen}_{D H C}\right) * \operatorname{Sen}_{E A} * \operatorname{Sen}_{W B}\right)}$} \\
\hline
\end{tabular}

Note: $A C$ Average Cost, DHC Alere Determine ${ }^{\mathrm{TM}}$ HIV-1/2 Ag/Ab Combo Test, EIA fourth generation enzyme immunoassay test, GP General Practitioner, $P$ Probability, Sen Sensitivity, Spec Specificity, WB Western Blot Test, + reactive test result, - nonreactive test result. $1=$ Medicare Rebate Item 36; $2=$ Medicare Rebate Item 69,384; 3 = Medicare Rebate Item 69,387; 4 = Medicare Rebate Item 44

the GP/doctor conventional setting because there are no follow-on visits for negative rapid results. There is, again, no loss of test effectiveness in the model due to the combination of conventional and POC testing being used for those with an initial reactive result.

Second, public SHC are far less costly (at less than half the cost) than conventional GP testing, largely due to the substantial salary savings of using nurses in combination with doctor/medical officers where the latter are only present for the event of a positive diagnosis.

Third, the peer rapid POC testing clinic is less expensive than almost all settings because it requires clients to only attend the clinic for an initial test overseen by a low cost peer tester, unless in the case of a reactive result when clients are referred for confirmatory testing and require a doctor/GP services. The standard rapid POC test results in a relatively small loss in test effectiveness. However, the average cost per test is significantly lower because of a combination of the lower peer salary levels compared to nurse and doctor/medical officers including GPs, and there is no need for a follow up visit to receive a result (either non-reactive or reactive) even when testing a comparatively higher prevalence MSM clientele. What primarily determines cost effectiveness of POC 
Table 4 Parallel Testing Regime: Community Peer Testing Service using Volunteer Peer Nurse Setting (Funded by Federal Government - Medicare and State Government)

\begin{tabular}{|c|c|c|c|c|c|c|c|c|}
\hline \multirow[b]{2}{*}{ Outcomes } & \multicolumn{4}{|c|}{ True HIV Positive $\left(P_{H I V}\right)$} & \multicolumn{4}{|c|}{ True HIV Negative $\left(1-P_{H I V}\right)$} \\
\hline & $\begin{array}{l}\mathrm{DHC}+ \\
\mathrm{EIA}+, \mathrm{WB}+\end{array}$ & $\begin{array}{l}\text { DHC-, EIA+, } \\
\text { WB+ }\end{array}$ & $\begin{array}{l}\text { DHC+, } \\
\text { EIA- }\end{array}$ & $\begin{array}{l}\text { DHC-, } \\
\text { EIA- }\end{array}$ & DHC+, EIA- & $\mathrm{DHC}+, \mathrm{EIA}+, \mathrm{WB}-$ & $\begin{array}{l}\text { DHC-, EIA+, } \\
\text { WB- }\end{array}$ & DHC-, EIA- \\
\hline $\begin{array}{l}\text { Probability of } \\
\text { result }\end{array}$ & $\begin{array}{l}\left(P_{H I V} *\right. \\
\operatorname{Sen}_{D H C} * \\
\operatorname{Sen}_{E I A} * \\
\left.\operatorname{Sen}_{W B}\right)\end{array}$ & $\begin{array}{l}\left(P_{H I V} *(1-\right. \\
\left.\operatorname{Sen}_{D H C}\right) * \\
\operatorname{Sen}_{E I A} * \\
\left.\operatorname{Sen}_{W B}\right)\end{array}$ & $\begin{array}{l}\left(P_{H I V} *\right. \\
\operatorname{Sen}_{D H C} \\
*(1- \\
\left.\left.\operatorname{Sen}_{E I A}\right)\right)\end{array}$ & $\begin{array}{l}\left(P_{H I V^{*}}\right. \\
(1- \\
\left.\operatorname{Sen}_{D H C}\right) \\
*(1- \\
\left.\left.\operatorname{Sen}_{E I A}\right)\right)\end{array}$ & $\begin{array}{l}\left(\left(1-P_{H I V}\right)\right. \\
*(1- \\
\left.\operatorname{Spec}_{D H C}\right) * \\
\left.\operatorname{Spec}_{E I A}\right)\end{array}$ & $\begin{array}{l}\left(\left(1-P_{H I V}\right) *(1-\right. \\
\left.\operatorname{Spec}_{D H C}\right) *(1- \\
\left.\left.\operatorname{Spec}_{E I A}\right) * \operatorname{Spec}_{W B}\right)\end{array}$ & $\begin{array}{l}\left(\left(1-P_{H I V}\right) *\right. \\
\operatorname{Spec}_{D H C} *(1- \\
\left.\operatorname{Spec}_{E I A}\right) * \\
\left.\operatorname{Spec}_{W B}\right)\end{array}$ & $\begin{array}{l}\left(\left(1-P_{H I V}\right) *\right. \\
\operatorname{Spec}_{D H C} * \\
\left.\operatorname{Spec}_{E I A}\right)\end{array}$ \\
\hline
\end{tabular}

Inputs

Visit 1: Initial Screening - Volunteer Peer Testing Facilitators

\begin{tabular}{|c|c|c|c|c|c|c|c|c|}
\hline Consult time & $20 \mathrm{~min}$ & $20 \mathrm{~min}$ & $20 \mathrm{~min}$ & $20 \mathrm{~min}$ & $20 \mathrm{~min}$ & $20 \mathrm{~min}$ & $20 \mathrm{~min}$ & $20 \mathrm{~min}$ \\
\hline $\begin{array}{l}\text { Staff Wage } \\
\text { (Rate/Hour): }\end{array}$ & $\$ 0$ & $\$ 0$ & $\$ 0$ & $\$ 0$ & $\$ 0$ & $\$ 0$ & $\$ 0$ & $\$ 0$ \\
\hline Staffing & $\begin{array}{l}\text { Volunteer } \\
\text { Nurse }\end{array}$ & $\begin{array}{l}\text { Volunteer } \\
\text { Nurse }\end{array}$ & $\begin{array}{l}\text { Volunteer } \\
\text { Nurse }\end{array}$ & $\begin{array}{l}\text { Volunteer } \\
\text { Nurse }\end{array}$ & $\begin{array}{l}\text { Volunteer } \\
\text { Nurse }\end{array}$ & Volunteer Nurse & Volunteer Nurse & $\begin{array}{l}\text { Volunteer } \\
\text { Nurse }\end{array}$ \\
\hline \multicolumn{9}{|l|}{ Pathology costs } \\
\hline $\begin{array}{l}\text { Rapid Alere } \\
\text { Determine Test }\end{array}$ & $\$ 11$ & $\$ 11$ & $\$ 11$ & $\$ 11$ & $\$ 11$ & $\$ 11$ & $\$ 11$ & $\$ 11$ \\
\hline ElA test & $\$ 15.65^{1}$ & $\$ 15.65^{1}$ & $\$ 15.65^{1}$ & $\$ 15.65^{1}$ & $\$ 15.65^{1}$ & $\$ 15.65^{1}$ & $\$ 15.65^{1}$ & $\$ 15.65^{1}$ \\
\hline WB & $\$ 29.00^{2}$ & $\$ 29.00^{2}$ & & & & $\$ 29.00^{2}$ & $\$ 29.00^{2}$ & \\
\hline
\end{tabular}

Visit 2: Test Results - Community Peer Organisation Bulk Billing GP Clinic

\begin{tabular}{|c|c|c|c|c|c|c|c|c|}
\hline Consult time & $40 \mathrm{~min}$ & 40 min & $20 \min$ & & $20 \mathrm{~min}$ & $20 \mathrm{~min}$ & $20 \min$ & \\
\hline $\begin{array}{l}\text { Medicate } \\
\text { Rebate }\end{array}$ & $\$ 105.55^{3}$ & $\$ 105.55^{3}$ & $\$ 71.70^{4}$ & & $\$ 71.70^{4}$ & $\$ 71.70^{4}$ & $\$ 71.70^{4}$ & \\
\hline Staffing & GP & GP & GP & & GP & GP & GP & \\
\hline $\begin{array}{l}\text { Total Cost of Each } \\
\text { Outcome }\end{array}$ & $\$ 161.20$ & $\$ 161.20$ & $\$ 98.35$ & $\$ 26.65$ & $\$ 98.35$ & $\$ 127.35$ & $\$ 127.35$ & $\$ 26.65$ \\
\hline $\begin{array}{l}\text { Average cost per } \\
\text { person tested }\end{array}$ & \multicolumn{8}{|c|}{ 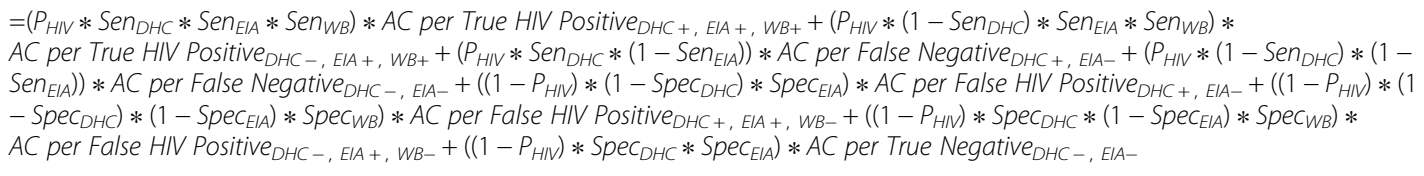 } \\
\hline $\begin{array}{l}\text { Average number } \\
\text { of tests for a } \\
\text { positive diagnosis }\end{array}$ & \multicolumn{8}{|c|}{$=\frac{1}{\left(P_{H N} * \operatorname{Sen}_{D H C} * \operatorname{Sen}_{E A} * \operatorname{Sen}_{W B}\right)+\left(P_{H N} *\left(1-\operatorname{Sen}_{D H C}\right) * \operatorname{Sen}_{E A} * \operatorname{Sen}_{W B}\right)}$} \\
\hline
\end{tabular}

Note: AC Average Cost, DHC Alere Determine ${ }^{\mathrm{TM}} \mathrm{HIV}-1 / 2 \mathrm{Ag} / \mathrm{Ab}$ Combo Test, EIA fourth generation enzyme immunoassay test, GP General Practitioner, $P$ Probability, Sen Sensitivity, Spec Specificity, WB Western Blot Test, + reactive test result, - nonreactive test result. $1=$ Medicare Rebate Item 69,$384 ; 2=$ Medicare Rebate Item 69,387; 3 = Medicare Rebate Item 44; 4 = Medicare Rebate Item 36

settings, is how inexpensive it is to screen negatives and not how expensive it is to diagnose positives.

\section{Sensitivity analysis}

This was conducted on variables of interest: HIV prevalence levels; sensitivity and specificity of the testing technologies as well as labour time and costs. No significant (>10\%) variation was evidenced in the cost results as a result of changes to any of the parameters.

\section{Discussion}

The cost results demonstrate that testing with rapid POC technologies, both peer facilitated and performed in the home/private, are the most cost effective means of achieving undiagnosed HIV diagnosis in WoP and MSM populations. This result holds irrespective of the losses in test effectiveness caused by the relatively lower sensitivity of the POC tests. Thus, in low prevalence countries like Australia, the biggest cost consideration for whole of population HIV screening should be how cheaply a screening modality can identify and diagnose negative cases, rather than how cheaply it can detect positive cases. Testing regimes that can screen but cannot confirm a diagnosis are highly cost effective. For example, community clinics using rapid POC testing can screen a negative case for under $\$ 35$ and confirm a positive case for less than $\$ 300$, whilst HIVST is dramatically less expensive, with screening of negative cases is of a 
Table 5 Point of Care Testing Regime: Community Peer Testing Service Setting (Funded by State Government)

\begin{tabular}{|c|c|c|c|c|c|c|}
\hline \multirow[b]{2}{*}{ Outcomes } & \multicolumn{3}{|c|}{ True HIV Positive $\left(P_{H I V}\right)$} & \multicolumn{3}{|l|}{ True HIV Negative $\left(1-P_{H I V}\right)$} \\
\hline & $\begin{array}{l}\text { DHC+, ElA+, } \\
\text { WB+ }\end{array}$ & DHC- & DHC+, EIA- & $\mathrm{DHC}+, \mathrm{EIA}+, \mathrm{WB}-$ & DHC+ EIA- & DHC- \\
\hline Probability of result & $\begin{array}{l}\left(P_{H I V} * \operatorname{Sen}_{D H C} *\right. \\
\left.\operatorname{Sen}_{E I A} * \operatorname{Sen}_{W B}\right)\end{array}$ & $\begin{array}{l}\left(P_{H I V} *(1-\right. \\
\left.\left.\operatorname{Sen}_{D H C}\right)\right)\end{array}$ & $\begin{array}{l}\left(P_{H I V} * \operatorname{Sen}_{D H C}\right. \\
\left.*\left(1-\operatorname{Sen}_{E I A}\right)\right)\end{array}$ & $\begin{array}{l}\left(\left(1-P_{H I V}\right) *\left(1-\operatorname{Spec}_{D H C}\right)\right. \\
\left.*\left(1-\operatorname{Spec}_{E I A}\right)+\text { Spec }_{W B}\right)\end{array}$ & $\begin{array}{l}\left(\left(1-P_{H I V}\right) *(1-\right. \\
\left.\left.\operatorname{Spec}_{D H C}\right) * \operatorname{Spec}_{E I A}\right)\end{array}$ & $\begin{array}{l}\left(1-P_{H I V}\right) \\
* \text { Spec }_{D H C}\end{array}$ \\
\hline \multicolumn{7}{|l|}{ Inputs } \\
\hline \multicolumn{7}{|c|}{ Visit 1: Initial Screening - Community Peer Testing Service } \\
\hline Consult time & $60 \mathrm{Min}$ & $30 \mathrm{Min}$ & $60 \mathrm{Min}$ & $60 \mathrm{Min}$ & $60 \mathrm{Min}$ & $30 \mathrm{Min}$ \\
\hline Staff Wage (Rate/Hour): & $\$ 45$ & $\$ 45$ & $\$ 45$ & $\$ 45$ & $\$ 45$ & $\$ 45$ \\
\hline Staffing & Peer & Peer & Peer & Peer & Peer & Peer \\
\hline \multicolumn{7}{|l|}{ Pathology costs } \\
\hline $\begin{array}{l}\text { Rapid Alere }(\mathrm{DHC}) \\
\text { Determine Test }\end{array}$ & $\$ 11$ & $\$ 11$ & $\$ 11$ & $\$ 11$ & $\$ 11$ & $\$ 11$ \\
\hline \multicolumn{7}{|c|}{ Referral to Bulk Billing GP for Confirmatory Testing } \\
\hline \multicolumn{7}{|l|}{ Visit 1: Initial Screening } \\
\hline Consult time & $20 \mathrm{~min}$ & & $20 \mathrm{~min}$ & $20 \mathrm{~min}$ & $20 \mathrm{~min}$ & \\
\hline Medicate Rebate & $\$ 71.70^{1}$ & & $\$ 71.70^{1}$ & $\$ 71.70^{1}$ & $\$ 71.70^{1}$ & \\
\hline Staffing & GP & & GP & GP & GP & \\
\hline \multicolumn{7}{|l|}{ Pathology costs } \\
\hline ElA test & $\$ 15.65^{2}$ & & $\$ 15.65^{2}$ & $\$ 15.65^{2}$ & $\$ 15.65^{2}$ & \\
\hline WB confirmatory & $\$ 29.00^{3}$ & & & $\$ 29.00^{3}$ & & \\
\hline \multicolumn{7}{|l|}{ Visit 2: Test Results } \\
\hline Consult time & $40 \mathrm{~min}$ & & $20 \mathrm{~min}$ & $20 \mathrm{~min}$ & $20 \mathrm{~min}$ & \\
\hline Medicate Rebate & $\$ 105.55^{4}$ & & $\$ 71.70^{1}$ & $\$ 71.70^{1}$ & $\$ 71.70^{1}$ & \\
\hline Staffing & GP & & GP & GP & GP & \\
\hline Total Cost of Each Outcome & $\$ 277.90$ & $\$ 33.50$ & $\$ 215.05$ & $\$ 244.05$ & $\$ 215.05$ & $\$ 33.50$ \\
\hline $\begin{array}{l}\text { Average cost per person } \\
\text { tested }\end{array}$ & \multicolumn{6}{|c|}{ 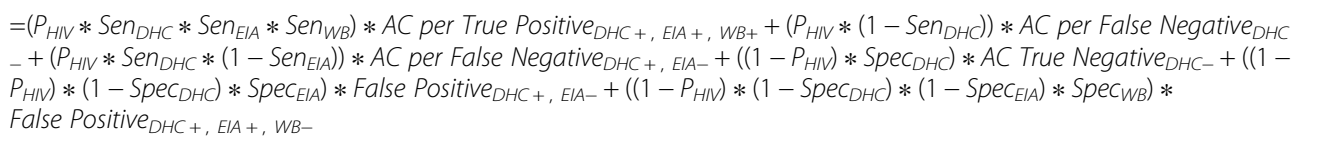 } \\
\hline $\begin{array}{l}\text { Average number of tests } \\
\text { for a positive diagnosis }\end{array}$ & $=\frac{1}{P_{H V V} * \operatorname{Sen}_{D H C} * \operatorname{Sen}_{E A A} * S E}$ & & & & & \\
\hline
\end{tabular}

Note: AC Average Cost, DHC Alere Determine ${ }^{\mathrm{Tm}}$ HIV-1/2 Ag/Ab Combo Test, EIA fourth generation enzyme immunoassay test, GP General Practitioner, $P$ Probability, Sen Sensitivity, Spec Specificity, WB Western Blot Test, + = reactive test result, $-=$ nonreactive test result. $1=$ Medicare Rebate Item $36 ; 2=$ Medicare Rebate Item 69,384; 3 = Medicare Rebate Item 69,387; 4 = Medicare Rebate Item 44

negligible cost especially with government supplied 'free' POC test kits.

Costs per diagnosis rise dramatically when prevalence is low [20]. Screening low-risk populations with more expensive and sensitive technologies is not cost-effective when prevalence is low [21]. Thus, it would make sense to use cheaper and less efficient (or effective) test technologies if cost is the only consideration. For example, results of this study suggests a HIV diagnosis made in a GP practice costs almost $\$ 900,000$, but a HIV diagnosis made in a MSM community clinic using POC costs less than $\$ 7000$.

The focus on cost per diagnosis in other studies often fails to provide a breakdown of what are the constituent drivers of cost or what were the factor inputs into the particular model (in this study these were prevalence, cost inputs and test effectiveness). Most studies either compare different testing regimes in the same clinical setting $[22,23]$ or the same regime in different clinic settings $[24,25]$. This study therefore provides an alternative costing model as it compares costs in different regimes and testing settings. The methodology applied in this study is important as HIV testing services are continually evolving to be delivered in different settings and by different people as testing technologies emerge and patterns of HIV infection, risk and population testing needs change [26]. The only comparative self-testing cost study is one which recently reported in the U.S. and has shown comparable values to those reported here [27]. The U.S. values were \$US61 per self-test 
Table 6 Point of Care Testing Regime: Home HIVST Setting (Funded by Consumer)

\begin{tabular}{|c|c|c|c|c|c|c|}
\hline \multirow[b]{2}{*}{ Outcomes } & \multicolumn{3}{|c|}{ True HIV Positive $\left(P_{H I V}\right)$} & \multicolumn{3}{|l|}{ True HIV Negative $\left(1-P_{H I V}\right)$} \\
\hline & $\begin{array}{l}\text { HIVST+, EIA+, } \\
\text { WB+ }\end{array}$ & HIVST - & HIVST +, EIA- & HIVST+, EIA+, WB- & HIVST+ EIA- & HIVST - \\
\hline Probability of result & $\begin{array}{l}\left(P_{H I V} * \operatorname{Sen}_{H I V S T} *\right. \\
\left.\operatorname{Sen}_{E I A} * \operatorname{Sen}_{W B}\right)\end{array}$ & $\begin{array}{l}P_{\text {HIV }} *(1- \\
\left.\operatorname{Sen}_{\text {HIVST }}\right)\end{array}$ & $\begin{array}{l}\left(P_{H I V} * \operatorname{Sen}_{H I V S T}\right. \\
\left.*\left(1-\operatorname{Sen}_{\text {EIA }}\right)\right)\end{array}$ & $\begin{array}{l}\left(\left(1-P_{H I V}\right) *\left(1-\text { Spec }_{H I V S T}\right)\right. \\
\left.*\left(1-\operatorname{Spec}_{E I A}\right)+\text { Spec }_{W B}\right)\end{array}$ & $\begin{array}{l}\left(\left(1-P_{H I V}\right) *(1-\right. \\
\left.\operatorname{Spec}_{H I V S T}\right) * \\
\left.\operatorname{Spec}_{E I A}\right)\end{array}$ & $\begin{array}{l}\left(1-P_{\text {HIV }}\right) \\
* \text { Spec }_{\text {HIVST }}\end{array}$ \\
\hline \multicolumn{7}{|l|}{ Inputs } \\
\hline \multicolumn{7}{|l|}{ Visit 1: Initial Screening } \\
\hline Staffing & Self & Self & Self & Self & Self & Self \\
\hline $\begin{array}{l}\text { OraQuick } \text { In-Home HIV } \\
\text { Test }\end{array}$ & $\$ 54.75$ & $\$ 54.75$ & $\$ 54.75$ & $\$ 54.75$ & $\$ 54.75$ & $\$ 54.75$ \\
\hline Postage & $\$ 7.50$ & $\$ 7.50$ & $\$ 7.50$ & $\$ 7.50$ & $\$ 7.50$ & $\$ 7.50$ \\
\hline \multicolumn{7}{|c|}{ Confirmatory Testing conducted at Bulk Billing GP } \\
\hline \multicolumn{7}{|l|}{ Visit 1: Initial Screening } \\
\hline Consult time & $20 \mathrm{~min}$ & & $20 \mathrm{~min}$ & $20 \mathrm{~min}$ & $20 \mathrm{~min}$ & \\
\hline Medicare Rebate & $\$ 71.70^{1}$ & & $\$ 71.70^{1}$ & $\$ 71.70^{1}$ & $\$ 71.70^{1}$ & \\
\hline Staffing & GP & & GP & GP & GP & \\
\hline \multicolumn{7}{|l|}{ Pathology costs } \\
\hline ElA test & $\$ 15.65^{2}$ & & $\$ 15.65^{2}$ & $\$ 15.65^{2}$ & $\$ 15.65^{2}$ & \\
\hline WB confirmatory & $\$ 29.00^{3}$ & & & $\$ 29.00^{3}$ & & \\
\hline \multicolumn{7}{|l|}{ Visit 2: Test Results } \\
\hline Consult time & $40 \mathrm{~min}$ & & $20 \mathrm{~min}$ & $20 \mathrm{~min}$ & $20 \mathrm{~min}$ & \\
\hline Medicare Rebate & $\$ 105.55^{4}$ & & $\$ 71.70^{1}$ & $\$ 71.70^{1}$ & $\$ 71.70^{1}$ & \\
\hline Staffing & GP & & GP & GP & GP & \\
\hline Total Cost of Each Outcome & $\$ 284.15$ & $\$ 62.25$ & $\$ 221.30$ & $\$ 250.30$ & $\$ 221.30$ & $\$ 62.25$ \\
\hline $\begin{array}{l}\text { Total Cost of Each Outcome } \\
\text { (excluding private costs) }\end{array}$ & $\$ 221.90$ & $\$ 0$ & $\$ 159.05$ & $\$ 188.05$ & $\$ 159.05$ & $\$ 0$ \\
\hline $\begin{array}{l}\text { Average cost per person } \\
\text { tested }\end{array}$ & \multicolumn{6}{|c|}{ 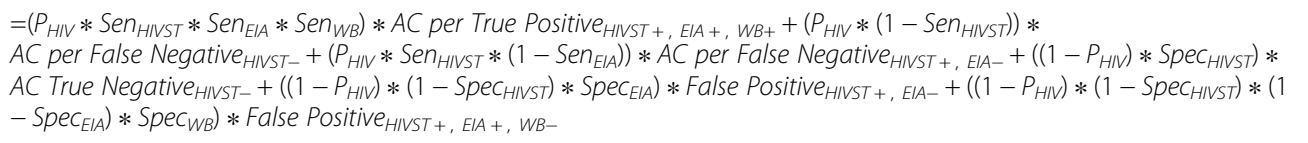 } \\
\hline $\begin{array}{l}\text { Average number of tests for } \\
\text { a positive diagnosis }\end{array}$ & \multicolumn{6}{|c|}{$=\frac{1}{P_{H I V} * \operatorname{Sen}_{H N S T} * \operatorname{Sen}_{E A} * \operatorname{Sen}_{W B}}$} \\
\hline
\end{tabular}

Note: AC Average Cost, DHC Alere Determine ${ }^{\mathrm{TM}}$ HIV-1/2 Ag/Ab Combo Test, ElA fourth generation enzyme immunoassay test, GP General Practitioner, $P$ Probability, Sen Sensitivity, Spec Specificity, WB Western Blot Test, + reactive test result, - nonreactive test result. $1=$ Medicare Rebate Item $36 ; 2=$ Medicare Rebate Item 69,384; 3 = Medicare Rebate Item 69,387; 4 = Medicare Rebate Item 44

completed; and an incremental cost per new HIV diagnosis of \$US9365 [27].

In this study a novel model has been presented by which to evaluate HIV testing regimes in a more flexible and easy manner than has been achieved previously. For example, decision tree models commonly use cost inputs or other bases of calculations which can be opaque. The model reported in this study identifies all possible outcomes and test results that a particular regime might lead to, and the cost inputs that these might incur, with population prevalence incorporated as a cost variable. This allows the cost of testing regime, settings and technologies to be established from a provider perspective. The cost incurred for a true positive diagnosis has been lowered by the adoption of less sensitive technologies, thus permitting a calculation of those choices in a trade-off between clinical intervention and efficiency and costs.

The results suggest the targeted use of POC testing technologies in communities and areas where prevalence might be higher than national average: e.g. MSM in Sydney, Melbourne and Brisbane can be done at extremely low cost with acceptable levels of effectiveness. Yet Australia has been slower than other countries at approving rapid testing technologies, in particular HIVST, despite an increased interest in testing using these devices [28-31]. HIVST is clearly less sensitive than either EIA or other rapid POC devices such as the DHC applied in this study as an initial screening tool, and will require, as with 
Table 7 Costs per Undetected HIV Diagnosis in the Whole of Population (WoP)

\begin{tabular}{|c|c|c|c|}
\hline \multirow{2}{*}{$\begin{array}{l}\text { Estimated Number of Undiagnosed Cases } \\
\text { Regime and Setting }\end{array}$} & \multicolumn{3}{|l|}{3350} \\
\hline & $\begin{array}{l}\text { Number of Tests Needed per Positive } \\
\text { Diagnosis }\end{array}$ & $\begin{array}{l}\text { Average Cost per Test } \\
\text { Regime } \\
\text { AUD\$ }\end{array}$ & $\begin{array}{l}\text { Total } \\
\text { Cost per } \\
\text { Positive } \\
\text { HIV } \\
\text { Diagnosis } \\
\text { AUD\$ }\end{array}$ \\
\hline \multicolumn{4}{|l|}{ Conventional Testing Regime } \\
\hline Private General Practice & 5602 & $\$ 159.10$ & $\$ 891,329$ \\
\hline Public Sexual Health Clinic & 5602 & $\$ 63.80$ & $\$ 357,411$ \\
\hline \multicolumn{4}{|l|}{ Parallel Testing Regime } \\
\hline Community Organisation (General Practice) & 5787 & $\$ 98.92$ & $\$ 572,542$ \\
\hline $\begin{array}{l}\text { Community Organisation (Peer Testing - } \\
\text { Volunteer) }\end{array}$ & 5787 & $\$ 27.14$ & $\$ 157,071$ \\
\hline \multicolumn{4}{|l|}{ Point of Care Testing Regime } \\
\hline Community Organisation (Peer Testing - Paid) & 5787 & $\$ 34.63$ & $\$ 200,436$ \\
\hline Home HIVST (include private costs) & 6111 & $\$ 62.32$ & $\$ 380,860$ \\
\hline Home HIVST (exclude private costs) & 6111 & $\$ 0.07$ & $\$ 416$ \\
\hline
\end{tabular}

all regimes and settings, confirmation of an initial reactive result by the conventional pathway to diagnosis. Even so, HIVST is comparatively very cost effective as a part of delivering an eventual positive diagnosis. Cost savings are further available due to the low numbers of clients who will need confirmatory testing. If the price of HIVST drops due to ease of availability or volume production, then the savings it presents as a means of testing are potentially large.

\section{Limitations}

This study found that the testing with rapid POC technologies are the most cost effective. However, there are two caveats to the results. First, the provability of having confirmation tests after Rapid POC testing regimes should be low; thus, the test efficiency could be lower and more costs might be required for the confirmation tests. Second, the number of false negative cases whilst not large would be sources of HIV transmission and might cause other costs.

Table 8 Costs per Undetected HIV Diagnosis in the MSM Population

\begin{tabular}{|c|c|c|c|}
\hline \multirow{2}{*}{$\begin{array}{l}\text { Estimated Number of Undiagnosed } \\
\text { Cases } \\
\text { Regime and Setting }\end{array}$} & \multicolumn{3}{|l|}{1848} \\
\hline & $\begin{array}{l}\text { Number of Tests Needed per Positive } \\
\text { Diagnosis }\end{array}$ & $\begin{array}{l}\text { Average Cost per Test } \\
\text { Regime } \\
\text { AUD\$ }\end{array}$ & $\begin{array}{l}\text { Total Cost per Positive HIV } \\
\text { Diagnosis } \\
\text { AUD\$ }\end{array}$ \\
\hline \multicolumn{4}{|l|}{ Conventional Testing Regime } \\
\hline Private General Practice & 165 & $\$ 159.46$ & $\$ 26,399$ \\
\hline Public Sexual Health Clinic & 165 & $\$ 64.62$ & $\$ 10,697$ \\
\hline \multicolumn{4}{|l|}{ Parallel Testing Regime } \\
\hline $\begin{array}{l}\text { Community Organisation (General } \\
\text { Practice) }\end{array}$ & 171 & $\$ 99.71$ & $\$ 17,053$ \\
\hline $\begin{array}{l}\text { Community Organisation (Peer Testing - } \\
\text { Volunteer) }\end{array}$ & 171 & $\$ 27.92$ & $\$ 4776$ \\
\hline \multicolumn{4}{|l|}{ Point of Care Testing Regime } \\
\hline $\begin{array}{l}\text { Community Organisation (Peer Testing - } \\
\text { Paid) }\end{array}$ & 171 & $\$ 36.01$ & $\$ 6159$ \\
\hline Home HIVST (include private costs) & 180 & $\$ 63.51$ & $\$ 11,469$ \\
\hline Home HIVST (exclude private costs) & 180 & $\$ 1.26$ & $\$ 227$ \\
\hline
\end{tabular}




\section{Conclusions}

The trade-off between test effectiveness and costeffectiveness may not necessarily be an area of charged medical ethical concern if POC testing is properly linked to pathways by which definitive diagnosis are given. This study advocates that a full suite of testing pathways including new and established testing technologies, especially POC, be employed in a manner that enables choices around testing and dispels barriers to testing for those at risk, and that new testing technologies should be deployed in an overall testing landscape that is costeffective and sensitive to client preferences for testing. As such, the relative cost of setting and technology deployed, though of clear importance to the health system, should not solely determine public health strategies geared to increasing HIV diagnosis.

\section{Supplementary Information}

The online version contains supplementary material available at https://doi. org/10.1186/s12913-020-06040-5.

Additional file 1: Table A1. Cost of Conventional Testing Regime: Private General Practice Clinic Setting - (Funded by Federal Government - Medicare). Table A2. Cost of Conventional Testing Regime: Public Sexual Health Clinic Setting (Funded by State Government). Table A3. Cost of Parallel Testing Regime: Community Peer Organisation Bulk Billing GP Clinic Setting (Funded by Federal Government - Medicare and State Government). Table A4. Cost of Parallel Testing Regime: Community Peer Testing Service using Volunteer Peer Nurse Setting (Funded by Federal Government - Medicare and State Government). Table A5. Cost of Point of Care Testing Regime: Community Peer Testing Service Setting (Funded by State Government). Table A6a. Cost of Point of Care Testing Regime: Home HIVST Setting (Includes Private Costs) (Funded by Consumer). Table A6b. Cost of Point of Care Testing Regime: Home HIVST Setting (Excludes Private Costs).

\section{Abbreviations}

ART: Antiretroviral treatment; DHC: Alere Determine ${ }^{\mathrm{TM}} \mathrm{HIV}-1 / 2 \mathrm{Ag} / \mathrm{Ab}$ Combo Test; EIA: Enzyme-linked immunosorbent assay; HIV: Human Immunodeficiency Virus; HIVST: HIV self-testing; GP: General Practice; MSM: Men who have sex with men; PLHIV: People living with HIV; POC: Point of care; SHC: Sexual Health Clinic; STI: Sexually transmissible infections; WB: Western Blot; WoP: Whole of population

\section{Acknowledgements}

The authors would like to acknowledgements the staff working within the clinics who willing volunteered to provide estimates of their clinic appointment times, salary structure details and testing pathways used as inputs in the development and analysis of this study's cost model.

\section{Authors' contributions}

All authors made substantial contributions to this work. Additionally, all authors contributed to drafting or revising this manuscript, and approved the final version for submission. OW designed the overall study and led the authorship of the paper. JD was involved in the conceptualisation of the study and led the data collection and development of the cost inputs, the literature review and data analysis process, and the initial write up of findings. AC developed the cost model, undertook the data collection and analysis and assisted with the initial write up of findings. CG was involved in the conceptualisation of the study, commented on the final draft, and approved the final version. JG reviewed and corrected the estimations and wrote the first and final draft of this manuscript. All authors read and approved the final manuscript.

\section{Funding}

This study was supported by a HIV Foundation Queensland Research and Program Grant. The HIV Foundation Queensland had no influence of any aspect of this research study nor approved the final manuscript.

\section{Availability of data and materials}

The datasets used and/or analysed during the current study are available from the corresponding author on reasonable request.

\section{Ethics approval and consent to participate}

This study was conducted under ethical approval from the University of Queensland Human Research Ethics Committee (Approval Number: 2017000159). Costing information and data was collected from community sites, sexual health and medical clinics. There were no individual participants and therefore no personal data was collected from individuals.

\section{Consent for publication}

Not applicable

\section{Competing interests}

The authors declare they have no competing interests

\section{Author details}

${ }^{1}$ School of Public Health, Faculty of Medicine, The University of Queensland, Herston, Australia. ${ }^{2}$ School of Medicine, Griffith University, Nathan, Australia. ${ }^{3}$ School of Commerce, University of Southern Queensland, Toowoomba, Queensland 4350, Australia. ${ }^{4}$ School of Accounting, Economics and Finance, University of KwaZulu-Natal, Durban, South Africa.

Received: 16 April 2020 Accepted: 21 December 2020 Published online: 07 January 2021

\section{References}

1. UNAIDS. 90-90-90 An ambitious treatment target to help end the AIDS epidemic. Geneva: Joint United Nations Programme on HIV/AIDS (UNAIDS); 2014.

2. Klepac P, Funk S, Hollingsworth TD, Metcalf CJE, Hampson K. Six challenges in the eradication of infectious diseases. Epidemics. 2015;10:97-101.

3. Kirby Institute. HIV, viral hepatitis and sexually transmissible infections in Australia. Annual Surveillance Report 2018. Sydney: The Kirby Institute, UNSW: The Kirby Institute, UNSW; 2018.

4. Bourne C, Allen D, Brown K, Davies SC, McNulty A, O'Connor CC, et al. What proportion of sexually transmissible infections and HIV are diagnosed in New South Wales' public sexual health services compared with other services? Sex Health. 2013;10(2):119-23.

5. Adias TC, Erhabor O. Human Immunodeficiency Virus Testing Algorithm in Resource Limiting Settings. In: Saxena SK, editor. Current Perspectives in HIV Infection. Rijeka: InTech; 2013. p. 480.

6. World Health Organisation. In: WHO, editor. Module 4: HIV Testing Strategies and Algorithms. Geneva: World Health Organization; 2005.

7. Mbachu II, Udigwe G, Joseph I, John O, Samuel UO, Joseph U, et al. The evaluation of accuracy of serial rapid HIV test algorithm in the diagnosis of HIV antibodies among pregnant women in south East Nigeria. BMC Res Notes. 2015;8(1):557

8. Adam PCG, de Wit JBF, Bourne CP, Knox D, Purchas J. Promoting regular testing: an examination of HIV and STI testing routines and associated socio-demographic, behavioral and social-cognitive factors among men who have sex with men in New South Wales, Australia. AIDS and Behavior. 2014;18(5):921-32

9. Bilardi JE, Walker S, Read T, Prestage G, Chen MY, Guy R, et al. Gay and bisexual Men's views on rapid self-testing for HIV. AIDS Behav. 2013;17(6): 2093-9.

10. Bolsewicz K, Debattista J, Vallely A, Whittaker A, Fitzgerald L. Factors associated with antiretroviral treatment uptake and adherence: a review. Perspectives from Australia, Canada, and the United Kingdom. AIDS Care. 2015;27(12):1429-38

11. Conway DP, Holt M, Couldwell DL, Smith DE, Davies SC, McNulty A, et al. Barriers to HIV testing and characteristics associated with never testing among gay and bisexual men attending sexual health clinics in Sydney. J Int AIDS Soc. 2015;18(1):20221-8. 
12. National HIV. Testing Policy Expert Reference Committee. National HIV Testing Policy 2017. Darlinghurst NSW: Commonwealth of Australia: Australasian Society for HIV Medicine (ASHM); 2017.

13. Chavez P, Wesolowski L, Patel P, Delaney K, Owen SM. Evaluation of the performance of the Abbott ARCHITECT HIV Ag/Ab combo assay. J Clin Virol. 2011;52:S51-S5

14. Rosenberg NE, Kamanga G, Phiri S, Nsona D, Pettifor A, Rutstein SE, et al. Detection of acute HIV infection: a field evaluation of the determine ${ }^{\bullet}$ HIV-1/ 2 Ag/Ab combo test. J Infect Dis. 2012;205(4):528-34.

15. Food and Drug Administration. In: FDA, editor. OraQuick In-Home HIV Test: summary of safety and effectiveness. United States: Food and Drug Administration; 2012

16. Australian Bureau of Statistics. 3101.0 - Australian Demographic Statistics. In: Australian Bureau of Statistics, editor. Canberra: Australian Bureau of Statistics; 2014.

17. Richters J, De Visser RO, Rissel C, Grulich A. Australian Study of Health and Relationships (ASHR) Sex in Australia 2 Summary. Melbourne: The Australian Research Centre in Sex, Health and Society (ARCSHS), La Trobe University; 2014.

18. The Kirby Institute. HIV, viral hepatitis and sexually transmissible infections in Australia Annual Surveillance Report 2015. Sydney: The Kirby Institute, University of NSW; 2015.

19. Williams OD, Dean JA, Harting K, Bath K, Gilks C. Implications of the on-line market for regulation and uptake of HIV self-testing in Australia. AIDS Care. 2016;29(1):112-7.

20. Hutchinson AB, Farnham PG, Sansom SL, Yaylali E, Mermin J. Costeffectiveness of frequent HIV testing of high-risk populations in the United States. J Acquir Immune Defic Syndr. 2016;71(3):323-30.

21. Castel AD, Choi S, Dor A, Skillicorn J, Peterson J, Rocha N, et al. Comparing cost-effectiveness of HIV testing strategies: targeted and routine testing in Washington, DC. PLoS One. 2015;10(10):e0139605.

22. Ekwueme DU, Pinkerton SD, Holtgrave DR, Branson BM. Cost comparison of three HIV counseling and testing technologies. Am J Prev Health. 2003; 25(2):112-21.

23. Farnham PG, Hutchinson AB, Sansom SL, Branson B. M. Comparing the costs of HIV screening strategies and technologies in health-care settings. Public Health Rep. 2008;123(Suppl 3):51-62.

24. Hoenigl M, Graff-Zivin J, Little SJ. Costs per diagnosis of acute HIV infection in community-based screening strategies: a comparative analysis of four screening algorithms. Clin Infect Dis. 2016;62(4):501-11.

25. Shrestha RK, Clark HA, Sansom SL, Song B, Buckendahl H, Calhoun CB, et al. Cost-effectiveness of finding new HIV diagnoses using rapid HIV testing in community-based organizations. Public Health Rep. 2008;123(SUPPL. 3):94100.

26. World Health Organisation. Consolidated guidelines on HIV testing services: 5Cs: Consent, Confidentiality. Counselling, Correct Results and Connection. Geneva: WHO; 2015.

27. Shrestha RK, Chavez P, Noble M, Sansom S, Sullivan P, Mermin J, et al. Estimating the costs and cost-effectiveness of HIV self-testing among men who have sex with men, United States. J Int AIDS Soc. 2020;23:e25445.

28. Bavinton BR, Brown G, Hurley M, Bradley J, Keen P, Conway DP, et al. Which gay men would increase their frequency of HIV testing with home selftesting. AIDS Behav. 2013;17(6):2084-92.

29. Chan D, Stewart M, Smith M, Price T, Lusk J, Ooi C, et al. The rise of targeted HIV oral rapid testing in Australia. Med J Aust. 2015;202(5):251-4.

30. Yan H, Zhang R, Wei C, Li J, Xu J, Yang H, et al. A peer-led, communitybased rapid HIV testing intervention among untested men who have sex with men in China: an operational model for expansion of HIV testing and linkage to care. Sex Transm Infect. 2014;90(5):388-93.

31. Yang M, Prestage G, Maycock B, Brown G, de Wit J, McKechnie M, et al. The acceptability of different HIV testing approaches: cross-sectional study among GMSM in Australia. Sex Transm Infect. 2014;90(8):592-5.

\section{Publisher's Note}

Springer Nature remains neutral with regard to jurisdictional claims in published maps and institutional affiliations.

\section{Ready to submit your research? Choose BMC and benefit from:}

- fast, convenient online submission

- thorough peer review by experienced researchers in your field

- rapid publication on acceptance

- support for research data, including large and complex data types

- gold Open Access which fosters wider collaboration and increased citations

- maximum visibility for your research: over $100 \mathrm{M}$ website views per year

At BMC, research is always in progress.

Learn more biomedcentral.com/submissions 